
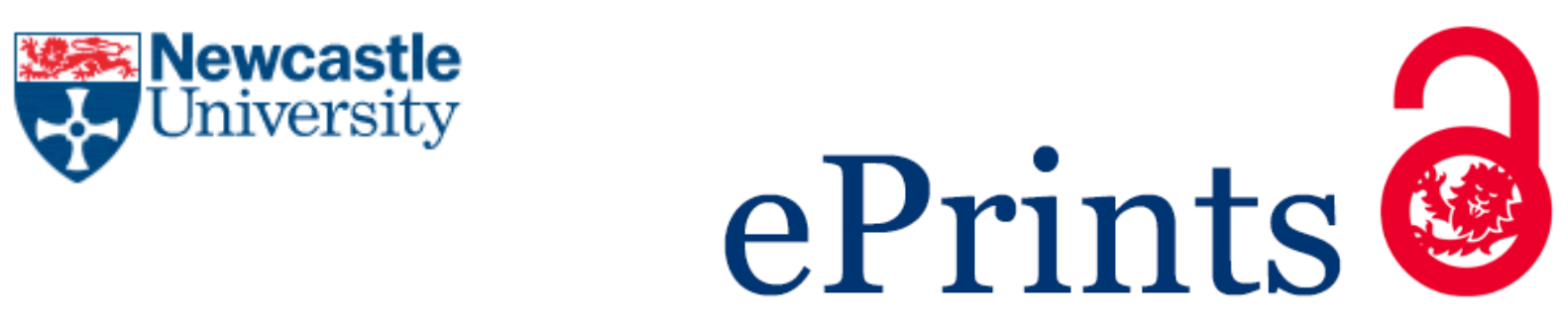

Grahame V, Brett D, Dixon L, McConachie H, Lowry J,

Rodgers J, Steen N, Le Couteur AS. (2015)

Managing Repetitive Behaviours in young children with Autism Spectrum Disorder (ASD): Pilot randomised controlled trial of a new parent group intervention.

Journal of Autism and Developmental Disorders

DOI: $10.1007 / s 10803-015-2474-x$

\title{
Copyright:
}

The final publication is available at Springer via http://dx.doi.org/10.1007/s10803-015-2474-x

Date deposited:

$01 / 06 / 2015$

Embargo release date:

03 June 2016

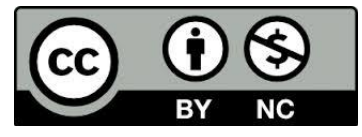

This work is licensed under a Creative Commons Attribution-NonCommercial 3.0 Unported License 
Managing Repetitive Behaviours in young children with Autism Spectrum Disorder (ASD): Pilot randomised controlled trial of a new Parent Group Intervention

\author{
Victoria Grahame ${ }^{1}$ \\ Denise Brett ${ }^{2}$ \\ Linda Dixon ${ }^{1}$ \\ Helen McConachie ${ }^{2}$ \\ Jessica Lowry ${ }^{2}$ \\ Jacqui Rodgers $^{3}$ \\ Nick Steen ${ }^{2}$ \\ Ann Le Couteur ${ }^{1,2}$
}

\begin{abstract}
${ }^{1}$ Northumberland, Tyne and Wear NHS Foundation Trust, UK
${ }^{2}$ Institute of Health and Society, Newcastle University, UK

${ }^{3}$ Institute of Neuroscience, Newcastle University, UK
\end{abstract}




\begin{abstract}
Early intervention for Autism Spectrum Disorder (ASD) tends to focus on enhancing socialcommunication skills. We report the acceptability, feasibility and impact on child functioning of a new 8 week parent-group intervention to manage restricted and repetitive behaviours (RRB) in young children with ASD aged 3-7 years. Forty-five families took part in the pilot RCT. A range of primary and secondary outcome measures were collected on four occasions (baseline, 10, $18 \& 24$ weeks) to capture both independent ratings and parent-reported changes in RRBs. This pilot established that parents were willing to be recruited and randomised, and the format and content of the intervention was feasible. Fidelity of delivery was high, and attendance was $90 \%$. A fully powered trial is now planned.
\end{abstract}

Keywords:, restricted and repetitive behaviour, parent group intervention, clinical global impression of improvement, randomised controlled trial

\title{
Correspondence to:
}

Dr Victoria Grahame

Complex Neurodevelopmental Disorders Service

Northumberland, Tyne and Wear NHS Foundation Trust

Walkergate Park

Benfield Road

Newcastle Upon Tyne

NE6 4QD

Tel: Tel: 0191287 5260/2

Email: victoria.grahame@ntw.nhs.uk 
Managing Repetitive Behaviours in young children with Autism Spectrum Disorder (ASD): Pilot randomised controlled trial of a new Parent Group Intervention

Autism spectrum disorder (ASD) is a common neurodevelopmental disorder that affects as many as $1 \%$ of children and has a considerable impact on a child's development (Baird et al. 2006). One key diagnostic domain of ASD is restricted, repetitive and stereotyped patterns of interests, behaviours and activities (ICD-10, World Health Organization, 1992; DSM-5, American Psychiatric Association, 2013). Narrowness of focus, inflexibility, perseveration of interest in activities and insistence on sameness reflect the restrictedness aspect of this domain, while repetition is demonstrated in repetitive speech, routines, rituals and rhythmic stereotypies (Leekam et al. 2011). The recent publication of DSM-5 criteria for a diagnosis of ASD includes sensory sensitivities within the restricted repetitive behaviour (RRB) domain for the first time. This new addition acknowledges the range of sensory symptoms experienced by individuals with ASD (Leekam et al. 2007).

The frequency and types of RRB displayed by children with ASD can vary according to age and cognitive ability. In a sample of 121 children with ASD, Militerni et al. (2002) found that children with ASD aged 2-4 years displayed more motor and sensory behaviours e.g. spinning or pulling coat zipper up and down while children aged 7-11 years displayed more complex behaviours such as insistence on sameness. They also found that children with lower IQ showed a more frequent interest in motor and sensory behaviours, with those with higher IQ demonstrating more complex RRB.. However not all RRB is regarded as problematic or warranting a targeted intervention. Some RRB may indicate an area of relative strength or a special skill . For some people, childhood circumscribed interests may lead to employment in adult life (Leekam et al. 2011). However, for the majority of individuals, RRB can have a disruptive impact on both the child and their family. RRB can interfere with the child's ability to learn new skills, engage in daily living activities and have been shown to take up large amounts of time (Cunningham \& Schreibman 2008; Dunlap et al. 1983; Rapp \& Vollmer 2005). RRB can also be stigmatizing (Cunningham \& Schreibman 2008), and lead to 
agitation or aggression if interrupted (Gordon 2000). Higher levels of RRB are also associated with heightened anxiety, for example, insistence on sameness, may be a maladaptive coping response to anxiety (Rodgers et al. 2012; Lidstone et al. 2014). RRB can interfere with family functioning and have been reported as among the most stressful behaviours for parents to manage (Bishop et al. 2007) in addition to provoking negative parenting styles (Greenburg at al. 2006).

An evidence base for the efficacy of parent-mediated interventions for young children with ASD is emerging (Oono et al 2013). Such interventions may enable parents to capitalise on teachable moments as they occur in the environment and provide learning opportunities during naturally occurring situations. Building on these real world experiences may also facilitate generalization of the child's learning across contexts (Carter et al. 2011). However, most ASD-specific early intervention programmes focus on social communication (Green et al 2010; Magiati et al. 2014; Oono et al. 2013). Parents rarely receive specific advice on their child's RRB and much of the existing evidence comes from single-case behavioural studies (Mulligan et al. 2014). Techniques used include differential reinforcement of variability in behavioural responding to reduce routines and insistence on sameness (Miller \& Neuringer 2000), response interruption and redirection for vocal and motor stereotypy (e.g. Ahrens et al. 2011), functional communication training for compulsive behaviour (Kuhn et al. 2009) and techniques such as visual schedules or video based modelling to help tolerate changes to routine or expand repetitive play (Hine \& Wolery 2006). While these studies offer evidence of success, limitations include implementation at specialised centres, highly individualised delivery with expert clinicians, and small sample sizes. One recent development of a parent-mediated intervention targeting RRB is the Family-Implemented Treatment for Behavioural Inflexibility (FITBI; Boyd et al. 2011). Direct instruction and naturalistic behavioural teaching methods were used to reduce targeted RRB, through a therapist working with parent and child for 12 weekly sessions for one to two hours. There was a reduction in the occurrence of RRB for the five child participants, and an increase in their engagement with more appropriate activities. However, as the intervention 
involved discrete teaching trials with the child during the weekly sessions, children displaying intermittent RRB were excluded as the behaviour could not be reliably elicited in the weekly session.

In acknowledging the unmet need for a parent-mediated intervention focussing on RRB, we have developed in collaboration with parents a new group based intervention to support parents to recognise, understand and manage RRB in young children with ASD. The Managing Repetitive Behaviours Programme (MRB $\odot$ ) is designed to be run by group leaders with experience working with young children with ASD. MRB@ utilises parent group learning alongside opportunities for mutual support and sharing of strategies. The aim is that through helping parents to gain knowledge and skills in managing their child's RRB, this would lead to improvement in the child's overall clinical function and reduction in RRB.

\section{Outcome measurement}

A wide range of measurement tools have been used in the study of RRB, mainly questionnaires and diagnostic interviews (Honey et al. 2012).. A limited range of structured observation methods focusing specifically on RRB have been developed. Watt et al. (2008) and Barber et al. (2012) investigated RRB in toddlers using a videotaped behaviour sample of the Communication and Symbolic Behaviour Scales Developmental Profile (CSBS-DP; Wetherby \& Prizant 2002). Boyd et al. (2010) developed the Direct Observation of Repetitive Behaviours in Autism (DOBRA) coding system which measures RRB, appropriate behaviour, problem behaviours, and interference. Harrop et al. (2014) developed a coding system using items from validated RRB questionnaires to measure RRB in toddlers and preschool children. However, the main limitation of direct observation in RRB is the failure to capture the range and variety of RRB.

An additional issue in measurement of change in RRB is the need for individualised measurement of outcomes. Arnold at al. (2003) proposed an individualised target symptom assessment procedure (target behaviour) involving quantified behaviour ratings of parent reports. 
Arnold and colleagues found this assessment highly convergent with their other outcome measures, evidence that individualised outcomes can reflect a true change in behaviour in heterogeneous groups.

This paper reports the findings of a feasibility and acceptability pilot randomised controlled trail of a new parent group intervention (Managing Repetitive Behaviours). The objectives of the study were to (i) investigate the feasibility and acceptability of the intervention, (ii) review the research protocol (including the randomisation and retention processes; and (iii) explore the utility of the outcome measures to assess the effects of the intervention on RRB, overall child functioning and parents' reported self-efficacy.

\section{Method}

\section{Participants}

Inclusion criteria for the study were parents of young children aged 3 years to 7 years 11 months, with a confirmed clinical diagnosis of ASD who identified a problematic RRB to work on during the group. Parents were required to have sufficient spoken English to take part in assessments and the group-based intervention, be willing to be randomised, agree to maintain current medication regime, and agree not to try any other new intensive interventions during the course of the study. There were no child exclusion criteria as the intervention is designed for parents of children with ASD across a range of abilities; from profound intellectual disability to average or above average intelligence. A total of 64 families expressed an interest in taking part; of these, two were excluded who did not meet the inclusion criteria (one child was too young, one parent did not have sufficient English) and 17 eventually declined to participate. Forty-five completed baseline assessments and progressed to randomisation (see Figure1), a sufficient number to estimate key parameters for a future trial (Julious, 2005; Lancaster et al., 2004).

\section{Measures}

\section{Baseline characterisation}


Autism Diagnostic Observation Schedule-2 (ADOS-2; Lord et al. 2012)

The ADOS-2 is a semi-structured observational assessment that involves social interaction between the examiner and the child; children were assessed with Modules 1, 2 or 3 according to language level and chronological age. The examiner scores elements of the child's behaviour in two domains, Social Affect and RRB. The scores for the domains are combined into a total score. Severity scores were also calculated according to Lord et al (2012) ranging from 1 to 10 , with scores of 1-2, 3-4, 5-7,8-10 indicating minimal to no evidence, low, moderate and high degree of autistic impairment, respectively. The ADOS 2 was conducted by trained research psychologists.

Social Responsiveness Scale (SRS; Constantino et al. 2005)

The SRS is a 65-item quantitative measure of the severity and type of social impairments that are characteristic of ASD, completed by the caregiver. Higher total scores on the SRS indicate greater severity of social impairment.

Vineland Adaptive Behaviour Scales II (VABS II; Sparrow, Cicchetti, \& Balla, 2005)

The VABS II measures aspects of an individual's level of adaptive functioning. The parent/caregiver rating form was used which focuses on four domains; communication, daily living skills, socialization and motor skills. The assessment was undertaken with parents as an interview by trained research psychologists. The domain composite scores provide an adaptive behaviour composite.

\section{Demographics}

In the baseline interview, parents were asked about their child age, gender, diagnosis and ethnicity; previous interventions, current medication and additional diagnoses. Information was also obtained on parents level of education, employment status, family structure, and if they had attended a previous course or intervention. Socioeconomic status was calculated using Townsend's Index of Deprivation based on the parent's postcode (Townsend, Phillmore, \& Beattle, 1988). 


\section{Primary Outcome Measures}

Taking into account the strengths and weaknesses of standardised questionnaire-based measures, observational techniques and individualised outcomes, the decision was made to include a combination of approaches in this pilot study to evaluate both RRB and impact on child and family functioning.

Clinical Global Impressions - Improvement scale (CGI-I; Guy, 1976)

The CGI-I requires the clinician to assess how much symptoms have improved or worsened relative to the child's baseline state using a 7-point scale (1 - very much improved; 2 - much improved; 3 minimally improved; 4 - no change; 5 - minimally worse; 6 - much worse; or 7 - very much worse). An independent panel of expert ASD clinicians, blind to group allocation, rated global improvement in overall interaction between parent and child and how much the child's RRB had changed over the 24 weeks since baseline. The clinicians rated independently using information from all time points (e.g. questionnaires, ADOS 2 videos, target behaviour vignettes and videos of parent child interactions) before reaching consensus. Ratings of 1 and 2 were regarded as 'improvement' at week 24.

\section{Measurement of RRB: Target Behaviour Vignette}

As part of the baseline characterisation, two repetitive behaviours of most concern for each parent/caregiver were identified and questions asked of the parent about their duration, impact and possible triggers and functions. The protocol for measuring change was developed by The Research Units on Paediatric Psychopharmacology and Psychosocial Interventions (RUPP Autism Network; Arnold et al. 2003). At each outcome assessment point, the parent was asked "At the beginning of the study you said you were concerned about (parent defined target behaviour at baseline). How has it been in the last couple of weeks?". The parent responses at each time point contributed to a written vignette by the researcher (who was blind to group intervention status). Identifying features such as 
gender and age were not included in each vignette. The baseline vignette detailing the target behaviour was paired with a vignette from each follow-up, to provide a comparison. In keeping with the procedure developed by Arnold et al (2003), after all data were collected, a panel of blinded autism researchers independently rated change in each target behaviour. Strong agreement was achieved (intraclass correlation coefficient $=0.81$ ). The average rating for each behaviour was used in analysis. The target behaviours were first categorised as: 1 - repetitive motor movements; 2rigidity, adherence to routine and insistence on sameness; 3 - preoccupation with restricted pattern of interest, limited play; or 4 - unusual sensory interests. Each pair of vignettes were rated on a 9 point scale of improvement/deterioration ( 1 - normal; 2 - markedly improved; 3 - definitely improved; 4 equivocally improved; 5 - no change; 6 - equivocally worse; 7-definitely worse; 8 - markedly worse; 9 - disastrously worse). Arnold et al (2003) defined a positive response as a rating of 3 or less and reported that the target behaviour measure was highly convergent with the CGI-I.

Measurement of RRB: Repetitive Behaviour Questionnaire - 2 (RBQ-2; Leekam et al., 2007).

The RBQ-2 is a 20-item questionnaire completed by parents/carers that measures the frequency/severity of RRB known to occur in both ASD and typical development. The RBQ-2 was developed using items from the RBQ (Turner, 1995) and the Diagnostic Interview for Social and Communication Disorders (DISCO; Wing et al., 2002). Leekam at al. (2007) found evidence for a four factor structure for the questionnaire aligned with the RRB described in the international classification systems for ASD; factor 1 - repetitive motor movements; factor 2- rigidity, adherence to routine and insistence on sameness; factor 3 - preoccupation with restricted pattern of interest; and factor 4 - unusual sensory interests. In a sample of typically developing two-year-olds, Leekam et al. (2007) found the RBQ-2 to have good internal consistency, inter-item validity, and across samples validity for the two geographical sub samples. Lidstone et al. (2014) also found the RBQ-2 to be a 
suitable measure of RRB in a sample of children with ASD aged 2-17 years, showing good internal consistency.

\section{Measurement of RRB: Parent-Child Interactions}

Parents and children were videoed interacting with a standardised set of toys for 10-minutes at baseline and at each outcome assessment. The frequency and duration of RRB observed, alongside parent strategies to manage these behaviours, were coded using a scheme previously developed by Shafi (2009; available on request from the corresponding author). The RRB coded were those likely to be observed in a short play interaction, and consequently are narrower and more detailed than the four categories described above. The types of RRB coded were: narrow repetitive interests; stereotyped behaviour/non-functional interests; specific sensory interests; unusual or repetitive motor movement; repetitive words/sounds. Parents' response to these RRB were categorised into four types: non-intervening; preventing; engaging; and distracting/developing. Ratings were undertaken by two blinded trained researchers. To assess inter-rater reliability, $25 \%$ of videos were double coded, half immediately after training and half during the coding process. Intraclass correlations for each of the categories of RRB and parent strategies ranged from 0.70 to 0.91 . Twelve videos did not have a total duration of ten-minutes; raters pro-rated these videos (dividing frequency and duration of each behaviour by the actual time of the video, and then multiplying by ten).

\section{Secondary Outcome Measures}

Parent self-efficacy (Sofronoff and Farbotko, 2002)

This 15 -item questionnaire completed by parents/carers measures behaviours typically exhibited by children with ASD including RRB. Parents indicated 'yes' or 'no' to whether the child displayed each of the behaviours in the previous month and then rated their confidence in managing the behaviours on a 6-point scale ranging from 0 (no confidence) to 5 (complete confidence). A mean self-efficacy 
score was then calculated by dividing the total confidence score by the number of behaviours reported as displayed.

\section{Teacher-completed $R B Q-2$}

The teacher of each child was also asked to complete the same 20 item Repetitive Behaviour Questionnaire - 2 (RBQ-2; Leekam et al., 2007) questionnaire as parents/carers to assess the frequency and severity of RRB in a school setting at each time point. This was to assess whether there was any difference in presentation of RRB between home and school and potential generalisation of results across settings.

\section{Focus groups}

Focus groups were held at the end of the trial and after data collection was complete. They were designed to discuss with parents three broad topics: the research process, acceptability of the MRB@ intervention and impact of the intervention on the participants, their children and the family. These groups were led by two independent facilitators with knowledge of ASD. Questions asked included 'How did you feel about being randomised?', 'How was your experience of being in the group?', and 'What impact has the MRB group had on your family's quality of life?'. Three focus groups with parents were completed, with between three and six parents attending each one. Framework analysis (Ritchie \& Spencer, 1994) was used to extrapolate main themes from the transcripts. Framework analysis begins deductively from the objectives set for the focus groups, but also uses an inductive approach from the accounts of the participants. i.e. new themes can emerge from the discussion with participants.

\section{Procedure}


A positive ethical opinion was received from Newcastle and North Tyneside Research Ethics Committee (11/NE/0379). Referrals were made through clinicians in Child and Adolescent Mental Health and early years education teams in North-East England who discussed the research with parents of children with ASD who met the inclusion criteria. They then returned expression of interest forms to the research team, who telephoned the families to arrange an initial home visit to take informed consent; baseline assessment was conducted in a clinical setting. Parents completed the RBQ-2, VABS II, SRS and parent self-efficacy. The RA conducted the target behaviour interview, ADOS 2 and video recorded the parent child interactions. Eligible participants were randomised in a 1:1 ratio to 'immediate intervention' or 'delayed intervention' within four blocks of referrals (www.randomization.com). Group interventions ran consecutively from May 2012 - May 2013 in four different locations. Assessments took place immediately at the end of the group intervention (10 weeks) and at two further time points, 18 weeks and 24 weeks after start of intervention. All outcome assessments included target behaviour vignette, parent RBQ-2, parent self-efficacy and parent-child interaction. Families remained under the clinical responsibility of local teams and continued to receive their existing routine care during the course of the study. At the end of the study the parents in the delayed group were offered the MRB intervention.

\section{Intervention}

The intervention involves eight weekly two hour sessions. The group is designed to be delivered by early years professionals with knowledge and experience of working with young children with ASD and their families. The intervention focuses on helping parents understand lower and higher order RRB, identify potential developmental and environmental factors that may trigger RRB for their child, and teaches parents to use a functional analytic approach to plan appropriate behavioural strategies which are effective for their child and family. Functional analysis helps parents to understand their child's RRB, where and how to intervene in order to manage this specific behaviour. Each parent is also given individual support [weeks $2 \& 6$ ] to further specify and review one of their chosen target RRBs. Parents video the target behaviour at home. This target behaviour is the focus for 
parents to practise the new skills they are learning, thus ensuring that strategies are individually tailored for each child, e.g. reinforcing other desirable behaviour.

The MRB parent group intervention has adapted and synthesised components of existing good practice for targeting and modifying RRB, challenging behaviour and social communication difficulties. It incorporates psychoeducation on ASD and RRB, helps parents formulate their child's behaviour using a basic functional analytic framework, facilitates group discussion and tailoring of strategies to manage RRB. The group also provides the opportunity to conceptualise RRB as a communicative function and therefore utilises adapted materials for communication (e.g. visual cues) and delivery techniques such as prompt, distract and redirect. It also includes original interactive activities developed in collaboration with parents such as quizzes. MRB places emphasis on discussion, developing and sharing ideas, experiences and strategies, importantly building parent's knowledge and confidence to manage their child's RRB.

\section{Fidelity}

Three independent raters were randomly allocated $50 \%$ of the recorded group intervention sessions to rate for fidelity to the treatment manual, using a checklist developed for the study. Raters rated fidelity to the manualised session content using a three point scale ( 0 - not at all; 1 - briefly covered but insufficiently; 2 - covered adequately), and therapeutic best practice including techniques used, generic acceptable therapeutic components, and undesirable components rated: 0 - not at all; 1 minimal evidence; 2 - several examples.

\section{Data Analysis}

Patterns of recruitment, retention and participation in the intervention were examined. Independent $t$ tests, chi square tests and Fisher's exact test were used to compare baseline scores on the 
characterisation and outcome measures. The target behaviour vignettes, parent RBQ-2, parent-child interactions, and self-efficacy were evaluated for completion rates and distribution of scores to assess appropriateness of parametric analysis. Transformations were used for the parent-child interactions; all other outcome measures had normal distribution. Repeated target behaviour vignettes, parent RBQ-2 and parental self-efficacy were analysed using a multi-level model (occasions nested within subjects), that is, first, whether a linear trend over time was an appropriate model and then whether the trend differed by allocation. Due to the number of separate codes (10), estimates of variability in parent-child interactions were based on 2 (group) x 4 (time-point) mixed analyses of variance (ANOVA). Groups were compared on the CGI-I using Fisher's exact test. For all analyses, a $p$ value of .05 was accepted as significant. Partial eta-squared $\left(\eta_{\mathrm{p}}{ }^{2}\right)$ statistic was used as a measure of effect size for ANOVA, where values $>0.01,0.06$, and 0.14 indicated a small, medium, and large effect size respectively (Cohen, 1988).

\section{Results}

\section{Participation and attrition}

Of the 25 families randomised to immediate intervention, one family did not attend the intervention, but completed follow-up assessments. Two families completed half of the sessions before dropping out of the course and research. For the delayed intervention group, two families were lost to follow-up at time point 2 (Figure 1). There was a completion rate of $89 \%$ for all families (from baseline to sixmonth follow-up). All results from previous outcome measures were carried forward for those who dropped out, in line with an intention to treat analysis. Of the 22 families who participated in the intervention, there was an average attendance rate of $90 \%$ at the sessions. Participant characteristics are shown in Table 1.

INSERT FIGURE 1 AND TABLE 1 ABOUT HERE

\section{Baseline equivalence of groups}


There was no difference between allocation groups on demographic variables or baseline characterisation of measures (See Tables 1,2).

\section{Fidelity of intervention}

A panel of expert observers rated adherence to the content of the 'Managing Repetitive Behaviours' (MRB) manual as $98 \%$ and delivery of therapeutic best practice as $93 \%$.

\section{Acceptability of Intervention}

\section{Focus Groups}

Each group followed a semi-structured topic guide with the aim of considering three key topics (i) the research process; (ii) acceptability of the MRB intervention; (iii) impact of the intervention on the participants, their children and the family.

\section{Research Process}

Parents' views about being part of a randomised controlled trial (RCT) were divided into four themes. Parents commented about (1) Assessments and follow-ups, (2) Contact with research team, (3) Randomisation and (5) Time in project. Discussions revealed some procedural issues with randomisation; some terms about randomisation might need to clarified and contact details of who to contact if there is an issue to do with randomisation need to be made more explicit in the future; " $I$ found it hard to not let it slip about taking part in the group." Some parents did not fully grasp what was meant by the term "the researchers are blind to who has attended the MRB programme" misinterpreting this to mean that they should conceal any new learning from researchers during the follow-up appointments.

\section{Acceptability of the Intervention}

Four principal themes emerged: (1) most participants reported that they had little knowledge of RRB before attending the intervention; (2) comments about the effort participants had made to attend and 
"get the most out" of the intervention; (3) positive comments about the intervention and the opportunity to "share experiences" with other parents who were "going through the same things" and (4) difficulties completing homework diaries or videoing their child engaging in a RRB although acknowledging it was an "opportunity for the professionals to see behaviour first hand and comment on it".

\section{Impact of the intervention on the participants, their children and the family}

Three themes relating to the impact of the MRB interventions were elicited: (1) positive changes that the course had had on them personally as participants such as feeling more confident, having an increased awareness and more knowledge about RRB and feeling more equipped to deal with their child's RRBs. "It has made me more aware of my actions and made me think about targeting other repetitive behaviours" (2) changes in their child's RRB in terms of frequency, duration or intensity, or that their child's behaviour was becoming more manageable: "The group has affected my child's RRB in an extraordinary way. He now knows he has boundaries within his RRB which was a huge issue for us" and (3) participants talked about their desire to disseminate strategies to other people involved their child's care, such as other family members and teachers. They frequently described wanting to get "everyone on the same page" in their family to provide a consistent approach to managing their child's RRB:

\section{Outcome measures}

Clinical Global Impressions - Improvement Scale

There was a significant difference between immediate intervention group (IG) and delayed intervention group (DG) on the blind rating of CGI-I (Fisher's exact test $\mathrm{p}=.05$, 2-tailed). Seven (30\%) participants in the IG met CGI-I criteria for very much improved/much improved (responders) compared to $1(5 \%)$ in the DG at time point (TP)3. There were $6(26 \%)$ in the IG and $4(20 \%)$ in the 
DG who met criteria for minimally improved, while $10(44 \%)$ in the IT and $15(75 \%)$ in the DG met the criteria for no change.

\section{Measurement of RRB: Target Behaviour Vignette}

From the boxplot, it can be seen that the immediate intervention group had greater improvement at each outcome assessment time point (i.e. lower rating; Figure 2). The trend across visits was fitted separately for immediate and delayed intervention groups. The immediate impact of the intervention characterised by the difference between the groups at the first outcome assessment visit was -0.45 (95\% CI: $-1.23,0.33)$. Then subsequently, the difference in the average change between visits was 0.13 (95\% CI: $-0.55,0.29$ ).

\section{INSERT FIGURE 2 ABOUT HERE}

To analyse the four factors of the target repetitive behaviours individually, participants in the immediate group were matched with participants from the delayed group based on category of RRB of their specific target behaviours (see Table 2). There was a significant change in ratings of the types of RRB included in Factor 3 (Preoccupations with restricted patterns of interest and limited play; $\mathrm{N}=$ 9 in immediate, $\mathrm{N}=8$ in delayed). Independent samples $t$-tests revealed a significant difference between groups at TP $2, t(15)=2.83, p=.01$, with the immediate group having greater improvement i.e. lower scores (median 2.25, interquartile range 1.63) than delayed (median 4.87, interquartile range 2.19). Similarly at TP 3 , the immediate group had greater improvement (median 3.00, interquartile range 1.88) than the delayed (median 5.12, interquartile range $0.88 ; t(15)=3.71, p=.002$.). Change in other factor behaviours (Repetitive motor movements; rigidity, adherence to routine and insistence on sameness; unusual sensory interests) was not significant.

\section{INSERT TABLE 2 ABOUT HERE}


The dependent variable, RBQ-2 total score at each time point was analysed using a mixed model with variation between parents and variation between time points included as random effects; baseline RBQ-2 was included as a covariate; and the difference between groups, a linear trend across time points together and their interaction were included as fixed effects. Maximum likelihood estimation procedures were used to generate interval estimates of the impact of the intervention. From the box plot, there was little evidence that the difference between groups changed over time (Figure 3).

\section{INSERT FIGURE 3 ABOUT HERE}

The total RBQ-2 score across all participants and occasions was 39.9 (95\% CI: 37.8, 42.0). The rate of change in immediate group was 0.07 units per time point (95\% CI: $-0.87,1.00)$. The rate of change in delayed group was -0.66 units per time point $(95 \%$ CI: $-1.71,0.38)$. The difference between these figures was 0.73 (95\% CI: -0.67, 2.14), indicating little evidence of a change over time in total RRB in either group or that the rate of change of total score differed between groups. Estimated difference between groups at first time point was -2.83 (95\% CI: -6.23, 0.57) not a clinically meaningful change. As can be seen in Table 3, Factor 2 'Rigidity, adherence to routine and insistence on sameness' appears to show a trend in the hypothesised direction; however, it was not statistically significant.

\section{INSERT TABLE 3 ABOUT HERE}

\section{Measurement of RRB: Parent Child Interactions}

A square root transformation was used to normalise the category 'stereotyped behaviour and nonfunctional interests', while a log transformation was used on the other categories of RRB, to allow parametric analysis. For one type of RRB 'stereotyped behaviour and non-functional interests' there was a significant main effect for time, $F(3,129)=3.35, p=.021, \eta_{\mathrm{p}}{ }^{2}=.072$ (a medium effect size) (see Table 2). There was also a reduction in the frequency of this RRB from baseline $(\mathrm{m}=4.91$, $\mathrm{sd}=4.04)$ to TP $2(\mathrm{~m}=2.93, \mathrm{sd}=2.97), t(44)=2.78, p=.008$, and from baseline to TP $3(\mathrm{~m}=2.98, \mathrm{sd}=3.76$, $t(44)=2.80, p=.008$ ), and a significant difference between groups at TP1, the immediate group had lower levels of this RRB at TP1 than the delayed group, $t(43)=2.47, p=.017$. Analysis of the 
individual parent strategies indicated a significant change in 'distracting/developing' strategy, as hypothesised. The data for this strategy was transformed using a square root transformation. The interaction was significant, $F(3,129)=2.82, p=.042 \eta_{\mathrm{p}}{ }^{2}=.062$ (a medium effect size). At TP2, there was an increase in the number of these strategies displayed in the immediate group, $t(43)=2.39, p=$ .021.

\section{Parent self-efficacy}

Self-efficacy was analysed by fitting a sequence of mixed models with time point scores nested within parents. Variation between parents and variation between time point scores within parents were included in the model as random effects with normal distribution. Baseline self-efficacy was included as a covariate. The difference between groups was included as a fixed effect. The boxplot of the raw data indicates the intervention had a positive effect on parent self-efficacy (Figure 4). There was a significant variation between parents $\sigma_{\mathrm{u}}=0.85(95 \% \mathrm{CI}: 0.68,1.07)$ and between outcome assessment visits within parents, $\sigma_{\mathrm{e}}=0.49$ (95\% CI: $\left.0.42,0.56\right)$. Baseline self-efficacy was a significant predictor of reported self-efficacy at follow-up outcome assessment visits. By including baseline selfefficacy, we explained some of the variation between parents, $\sigma_{\mathrm{u}}$ decreased to 0.71 (95\% CI: 0.56 , 0.90). Fitting an impact of the intervention, our results suggest that the intervention increased selfefficacy by 0.74 (95\% CI: $0.34,1.14)$.

\section{INSERT FIGURE 4 ABOUT HERE}

\section{Teacher $R B Q-2$}

There were no significant changes over time in teacher-rated total RBQ-2 scores or factors of the teacher RBQ-2. However not all RRB behaviours identified by parents are likely to occur at school e.g. rigid restricted bath time routine. 


\section{Discussion}

To our knowledge, this is the first randomized controlled trial of a parent group intervention designed to help parents recognise, understand and manage RRB in young children with ASD. Results from this pilot trial support the feasibility and acceptability of both this new intervention, and the recruitment and research procedures. This pilot trial established that families were willing to be recruited and randomised, parents found the format and content of the intervention acceptable and attrition was small. Group leaders were able to deliver the programme in a consistent fashion as evidenced by a high degree of fidelity to the treatment manual. The properties of the outcome measures have also been assessed.

The study was not designed as a fully powered trial. However, there is preliminary evidence that this new MRB $\odot$ parent-group intervention led to greater gains in parent self-efficacy and improvement in overall interaction between parent and child (CGI-I). Although 30\% of children in the intervention group being classified as definitely 'improved' at 6 months may appear somewhat low, nevertheless another $26 \%$ made some improvement, and for families any change in difficult-tomanage behaviours may be of clinical significance; $75 \%$ of the delayed group were observed to make no change at all.

There is also a suggestion that for parents in the intervention group who reported their chosen target RRB to be of the category 'preoccupations with restricted patterns of interest and limited play' (Factor 3), there was evidence of a significant improvement in independently-rated target behaviour vignettes compared to the delayed group. Equipping parents with environmental strategies in which they can engage, distract or develop their child's RRB may have more utility when the target behaviours of concern are rigidity, routines or preoccupations. Perhaps behaviours that may have a cognitive component or be more likely to be related to executive control, may be the most appropriate 
for parents to attempt to address, compared to some of the repetitive sensorimotor behaviours that may represent separate neurological pathways regulated by internal sensory mechanisms.

MRB is a parent-implemented intervention. Central to its ethos is the assumption that parents can increase their understanding and management of RRB which in turn will build parents' confidence in their parenting skills. This study reports improvement in parent self-efficacy that was maintained at 6-month outcome measurement. Given that repetitive behaviours are reported to be some of the most stressful of ASD behaviours to manage (Bishop et al. 2007), it is a strength of this parent-group intervention that parents reported large gains in confidence in managing their child's RRB.

It is possible that teaching parents how to consider RRB using a functional analytic framework approach enabled parents of young children with ASD to both consider the communicative function of their child's RRB and implement tailored strategies to manage them, which in turn may have led to the improved outcomes reported.

Utility of outcome measures

A strength of the study is that RRB and the possible impact of the intervention were measured in several different ways. The CGI-I (a 'blind' independent measure regularly used in the evaluation of medication and other interventions in child mental health research) showed promise as an appropriate overall outcome measure for detecting treatment response.

Three different approaches to the measurement of RRB were included in this study. In line with previous research (Leekam et al 2007; Lidstone et al. 2014), the RBQ-2 was found to be an acceptable measure for use with parents of children with ASD. However the use of a total score of RRBs was not apparently sensitive to change. Despite this limitation, the parent RBQ2 may have utility in future studies exploring generalisation over time. Teachers were also asked to complete the RBQ2, to provide a measure of RRB in a different setting. In the previous development work, parents had identified the importance of recording the levels of and any changes in RRB in other social contexts 
(such as nursery/education setting). Teachers were able to complete the teacher-version of the RBQ-2 but indicated that some items were not relevant to the education setting (e.g. 'insist on wearing the same clothes or refuses to wear new clothes). Education staff were also aware that they may not see the same types of RRB that parents reported in the home. In the development of therapeutic interventions for children with ASD, the recognition of the difficulties many children experience transferring newly acquired skills from one setting to another (generalisation) is widely recognised and reported (e.g. Parsons \& Mitchell 2002; Swettenham, 1996 ; Green et al. 2010). A recently completed report on outcome measures has also highlighted the need for a questionnaire appropriate for young children with ASD which can be used to measure RRB across settings (McConachie et al 2015)

The target behaviour vignette was practical, reliably rated, and sensitive to change. One important advantage of this outcome measure is that it captures the idiographic account of the particular behaviour that is most relevance to the family, whilst at the same time the vignette rating procedure provides a method for obtaining quantitative data (change score) independent of parent bias and judged by 'blind' expert raters. The results are suggestive of an impact of the intervention in particular for rigidity of routines and preoccupations leading to limited opportunities for play (Factor 3) (Hine \& Wolery 2006; Honey et al 2007).

Turning to the parent child interactions, although this measure provided a way of directly observing RRB that could be blind-rated, it is unlikely that for each child the behaviours of concern would be elicited in a ten-minute play interaction. This measure appears to be most useful with directly observable RRB such as stereotypical motor movements and sensory behaviours and less so with preoccupations, routines and sameness. The parent self-efficacy scale was found to be an appropriate 
measure suitable for this young, ASD population and their parents. In this study the measure identified a clear treatment effect for those in the immediate intervention group.

Limitations

The major limitation of the current study is sample size; although it was adequate for a phase-II pilot, definitive conclusions about efficacy of the intervention cannot be drawn from the data.

Implications for further research

This new parent group intervention MRB, has been developed in collaboration with parents of young children with ASD utilising an 'active' research methodology. It is a psychoeducational intervention that incorporates evidence based knowledge of ASD, the principles of a functional analysis approach to understanding behaviours combined with the mutual support of group peer learning. Parent involvement has informed every aspect of the design of the intervention, from training materials to the acceptability of the devised programme, piloting the feasibility of outcome measures and the use of video feedback as a strategy for working on an agreed target RRB, through to contributing to the design of the research protocol. This collaborative 'active' research model of working jointly with parents has in the opinion of all those involved in this study been of great mutual benefit. Parent involvement has brought new perspectives and helped ensure that both the development of the intervention and the research is relevant to the concerns and needs of parents and families of young children with ASD. Planning research into complex interventions such as parent-mediated interventions should address service user research priorities; this can be facilitated through adoption of an 'active' research model. Future research also needs to identify a reliable measure of observable $\mathrm{RRB}$, capturing all topographies of RRB, across a range of ages and cognitive abilities. Promising 
results have been found using the DOBRA coding system (Boyd et al. 2010); however, implementation of this coding system needs to occur in a larger, fully powered study to assess its sensitivity. It is desirable that any intervention to target RRB should consider how best to work across different social contexts (home, educational and other setting); this will also require a tool to measure change in RRBs across settings.

\section{Conclusion}

This study has shown that the Managing Repetitive Behaviours: MRB $\odot$ programme was acceptable to parents and feasible to implement. The estimates of variability in outcome measures indicated large gains in parental self-efficacy and suggestive improvements in overall functioning for the child including an improvement in the target RRB in the immediate intervention group. A multi-site trial is now required to establish the efficacy of this intervention, which should be powered to examine potential moderators and mediators of treatment effects.

This paper describes independent research commissioned by the National Institute for Health Research (NIHR) under the Research for Patient Benefit programme (PB-PG-1010-23305). The views expressed are those of the authors and not necessarily those of the NHS, the NIHR or the Department of Health. 


\section{References}

Ahrens, E. N., Lerman, D. C., Kodak, T., Worsdell, A. S., \& Keegan, C. (2011). Further evaluation of response interruption and redirection as treatment for stereotypy. Journal of Applied Behavior Analysis, 44, 95-108. doi:10.1901/jaba.2011.44-95

American Psychiatric Association (2013). Diagnostic and statistical manual of mental disorders (5th edition). Washington, DC.

Arnold, L. E., et al. (2003). Parent-defined target symptoms respond to risperidone in RUPP autism study: customer approach to clinical trials. Journal of the American Academy of Child and Adolescent Psychiatry, 42, 1443-50. doi:10.1097/00004583-200312000-00011

Baird, G., Simonoff, E., Pickles, A., Chandler, S., Loucas, T., Meldrum, P., \& Charman, T. (2006). Prevalence of disorders of the autism spectrum in a population cohort of children in South Thames: the Special Needs and Autism Project (SNAP). Lancet, 368, 210-5. doi:10.1016/S0140-6736(06)69041-7

Barber, A. B., Wetherby, A. M., \& Chambers, N. W. (2012). Brief report: Repetitive behaviors in young children with autism spectrum disorder and developmentally similar peers: A follow up to Watt et al. (2008). Journal of Autism and Developmental Disorders, 42 , 2006-2012

Bishop, S. L., Richler, J., Cain, A. C., \& Lord, C. (2007). Predictors of perceived negative impact in mothers of children with autism spectrum disorder. American Journal of Mental Retardation, 112, 450-61. doi:10.1352/0895-8017(2007)112[450:POPNII]2.0.CO;2

Boyd, B. A., McDonough, S. G., Rupp, B., Khan, F., \& Bodfish, J.W. (2011). Effects of a familyimplemented treatment on the repetitive behaviors of children with autism. Journal of Autism and Developmental Disorders, 41, 1330-41. doi:10.1007/s10803-010-1156-y

Boyd, B. A., Rupp, B., \& Bodfish, J. W. (2010). Direct observation of repetitive behaviors in autism. University of North Carolina at Chapel Hill. Unpublished manual.

Carter, A. S., Messinger, D. S., Stone, W. L., Celimli, S., Nahmias, A. S., \& Yoder, P. (2011). A randomized controlled trial of Hanen's 'More Than Words' in toddlers with early autism 
symptoms. Journal of Child Psychology and Psychiatry, and Allied Disciplines, 52, 741-52. doi:10.1111/j.1469-7610.2011.02395.x

Constantino, J. N., \& Gruber, C. P. (2005). Social Responsiveness Scale (SRS). Los Angeles, CA: Western Psychological Services.

Cohen, J. (1988). Statistical power analysis for the behavioral sciences. Hillsdale, New Jersey: Lawrence Erlbaum Associates.

Cunningham, A. B., \& Schreibman, L. (2008). Stereotypy in autism: The importance of function. Research in Autism Spectrum Disorders, 2, 469-479. doi:10.1016/j.rasd.2007.09.006

Dunlap, G., Dyer, K., \& Koegel, R. L. (1983). Autistic self-stimulation and intertrial interval duration. American Journal of Mental Deficiency, 88, 194-202.

Gordon, C. T. (2000). Commentary: Considerations on the pharmacological treatment of compulsions and stereotypies with serotonin reuptake inhibitors in pervasive developmental disorders. Journal of Autism and Developmental Disorders, 30, 437-438.

Green, J., Charman, T., McConachie, H., Aldred, C., Slonims, V., Howlin, H., Le Couteur, A., Leadbitter, K., Hudry, K., Byford, S., Barrett, B.,Temple, K., Macdonald, W., Pickles, A., and the PACT Consortium. (2010). Parent-mediated communication-focused treatment in children with autism (PACT): a randomised controlled trial. The Lancet, 375(9732), 2152-2160. eScholarID:81710 | DOI:10.1016/S0140-6736(10)60587-9

Greenberg, J. S., Seltzer, M. M., Hong, J., \& Orsmond, G. I. (2006). Bidirectional effects of expressed emotion and behavior problems and symptoms in adolescents and adults with autism. American Journal of Mental Retardation, 111, 229-49. doi:10.1352/08958017(2006)111[229:BEOEEA]2.0.CO;2

Guy, W. (Ed. 1976). Clinical Global Impression. In: ECDEU Assessment Manual for Psychopharmacology, revised ( pp 218-222). National Institute of Mental Health. Rockville MD. 
Harrop, C., McConachie, H., Emsley, R., Leadbitter, K., \& Green, J. (2014). Restricted and repetitive behaviors in autism spectrum disorders and typical development: Cross-sectional and longitudinal comparisons. Journal of Autism and Developmental Disorders, 44,5, 1207-1219.

Hine, J. \& Wolery, M. (2006). Using point-of-view video modeling to teach play to preschoolers with autism. Topics in Early Childhood Special Education (26)2, 83-93.

Honey, E., Rodgers, J., \& McConachie, H. (2012). Measurement of restricted and repetitive behaviour in children with autism spectrum disorder: Selecting a questionnaire or interview. Research in Autism Spectrum Disorders, 6, 757-776. doi:10.1016/j.rasd.2011.10.011

Honey, E., Leekam, S., McConachie, H., \& Turner, M.(2007).Repetitive Behaviour and Play in Typically Developing Children and Children with Autism Spectrum Disorders. Journal of Autism and Developmental Disorders, 37(6), 1107-15

Julious, S. A. (2005) Sample size of 12 per group rule of thumb for a pilot study. Pharmacentical Statistics, 4, 287-291.

Kuhn, D. E., Hardesty, S. L. \& Sweeney, N. M. (2009). Assessment and treatment of excessive straightening and destructive behaviour in an adolescent diagnosed with autism. Journal of Applied Behavior Analysis, 42, 355-60. doi:10.1901/jaba.2009.42-355

Lancaster, G. A., Dodd, S., \& Williamson, P. R. (2004). Design and analysis of pilot studies: recommendations for good practice. Journal of Evaluation in Clinical Practice, 10, 307-12. doi:10.1111/j.2002.384.doc.x

Leekam, S. R., Prior, M. R., \& Uljarevic, M. (2011). Restricted and repetitive behaviors in autism spectrum disorders: a review of research in the last decade. Psychological bulletin, 137, 56293. doi:10.1037/a0023341

Leekam, S., Tandos, J., McConachie, H., Meins, E., Parkinson, K., \& Wright, C., et al. (2007). Repetitive behaviours in typically developing 2-year-olds. Journal of Child Psychology and Psychiatry, and Allied Disciplines, 48, 1131-8. doi:10.1111/j.1469-7610.2007.01778.x 
Leekam, S. R., Nieto, C., Libby, S. J., Wing, L., \& Gould, J. (2007). Describing the sensory abnormalities of children and adults with autism. Journal of Autism and Developmental Disorders, 37, 894-910. doi:10.1007/s10803-006-0218-7

Lidstone, J., Uljarevic, M. (joint first authors), Sullivan, S., Rodgers, J., McConachie, H., Freeston, M., et al. (2014). Relations among restricted and repetitive behaviours, anxiety and sensory features in children with autism spectrum disorder. Research in Autism Spectrum Disorders, $8(2), 82-92$.

Lord, C., Rutter, M., DiLavore, P. C., et al. (2012). Autism diagnostic observation schedule, second edition: ADOS-2. Torrance: Western Psychological Services.

Magiati, I., Tay, X. W., \& Howlin, P. (2014). Cognitive, language, social and behavioural outcomes in adults with autism spectrum disorders: A systematic review of longitudinal follow-up studies in adulthood. Clinical Psychology Review, 34, 73-86. doi:10.1016/j.cpr.2013.11.002

McConachie, H., Randle, V., Hammal, D., \& Le Couteur, A. (2005). A controlled trial of a training course for parents of children with suspected autism spectrum disorder. The Journal of Pediatrics, 147, 335-40. doi:10.1016/j.jpeds.2005.03.056

McConachie, H., Parr, J., M Glod, M., Hanratty, J., Livingstone, N., Oono, I.P., Robalino, S., Baird, G., Beresford, B., Charman, T., Garland, D., Green, J., Gringras, P., Jones, G., Law, J., Le Couteur, A., Macdonald, G., McColl, E.M., Morris, C., Rodgers, J., Simonoff, E., Terwee, C.B., Williams, K. (2014). Systematic review of tools to measure outcomes for young children with autism spectrum disorder. HTA Journal (in press)

Militerni, R., Bravaccio, C., Falco, C., Fico, C., \& Palermo, M. T. (2002). Repetitive behaviors in autistic disorder. European Child \& Adolescent Psychiatry, 11, 210-8. doi:10.1007/s00787002-0279-x

Miller, N., \&Neuringer, A. (2000). Reinforcing variability in adolescents with autism. Journal of Applied Behavior Analysis, 33, 151-65. doi:10.1901/jaba.2000.33-151 
Mulligan, S., Healy, O., Lydon, S., Moran, L., \& Foody, C. (2014). An Analysis of Treatment Efficacy for Stereotyped and Repetitive Behaviors in Autism. Journal Autism Developmental Disorders, 1, 143-164.

Oono, I. P., Honey, E. J., \& McConachie, H. (2013). Parent-mediated early intervention for young children with autism spectrum disorders (ASD). The Cochrane Database of Systematic Reviews 4, CD009774. doi:10.1002/14651858.CD009774.pub2

Parsons, S., \& Mitchell, P. (2002). The potential of virtual reality in social skills training for people with autistic spectrum disorders. Journal of Intellectual Disability Research, 46, 430-43.

Rapp, J.T.,Vollmer, T. R. (2005). Stereotypy I: a review of behavioral assessment and treatment. Research in Developmental Disabilities, 26, 527-47. doi:10.1016/j.ridd.2004.11.005

Ritchie, J., \& Spencer, L. (1994) Qualitative data analysis for applied policy research. In A. Bryman and R. G. Burgess (Eds), Analysing Qualitative Data (pp. 173-194). London: Routledge.

Rodgers, J., Glod, M., Connolly, B., \& McConachie, H. (2012). The relationship between anxiety and repetitive behaviours in autism spectrum disorder. Journal of Autism and Developmental Disorders, 42, 2404-9. doi:10.1007/s10803-012-1531-y

Shafi, D. (2009). How parents of young children with autism manage repetitive behaviour: an observational study. Unpublished Clin.Psy.D. thesis, Newcastle University.

Sofronoff, K., \& Farbotko, M. (2002). The effectiveness of parent management training to increase self-efficacy in parents of children with Asperger syndrome. Autism: the International Journal of Research and Practice, 6, 271-86.

Sparrow, S. S., Cicchetti, V. D., \& Balla, A. D. (2005). Vineland adaptive behavior scales. 2nd edition. Circle Pines, MN: American Guidance Service.

Swettenham, J. (1996). Can children with autism be taught to understand false belief using computers? Journal of Child Psychology and Psychiatry, and Allied Disciplines, 37, 157-65.

Townsend, P., Phillimore, P., \&Beattie, A. (1988). Health and deprivation: inequality and the North. London: Croom Helm. 
Turner, M. (1995). Repetitive behaviour and cognitive functioning in autism. Unpublished PhD thesis, University of Cambridge.

Watt, N., Wetherby, A. M., Barber, A., \& Morgan, L. (2008). Repetitive and stereotyped behaviors in children with autism spectrum disorders in the second year of life. Journal of Autism and Developmental Disorders, 38, 1518-33. doi:10.1007/s10803-007-0532-8

Wetherby, A., \& Prizant, B. (2002). Communication and symbolic behavior scales developmental profile-First normed edition. Baltimore, MD: Paul H. Brookes

Winburn, E., Charlton, J., McConachie, H., McColl, E.,, Parr, J., O’Hare, A. et al (2014). Parents' and child health professionals' attitudes towards dietary interventions for children with autism spectrum disorders. Journal of Autism and Developmental Disorders, 44, 747-757.

Wing, L., Leekam, S. R., Libby, S. J., Gould, J. \& Larcombe, M. (2002). The Diagnostic Interview for Social and Communication Disorders: background, inter-rater reliability and clinical use. Journal of Child Psychology and Psychiatry, and Allied Disciplines, 43, 307-25.

World Health Organisation (1992). International Classification of Diseases (ICD 10. Geneva: World Health Organisation. 
Table1. Participant Demographics

\begin{tabular}{|c|c|c|c|}
\hline & Immediate $(\mathrm{N}=25)$ & Delayed $(\mathrm{N}=20)$ & Difference \\
\hline & $(\%)$ & (\%) & $p$ \\
\hline \multicolumn{4}{|l|}{ Child } \\
\hline Age (months) & $60.44 \mathrm{M}(13.44 \mathrm{SD})$ & $62.75 \mathrm{M}(16.89 \mathrm{SD})$ & $.612 *$ \\
\hline Gender & 24 male, 1 female & 15 male, 5 female & $.074 \dagger$ \\
\hline Psychotropic Medication & 0 male, 1 female & 0 male, 0 female & $.444 \dagger$ \\
\hline Melatonin & 3 male, 1 female & 3 male, 1 female & $1.0 \dagger$ \\
\hline Child's Ethnicity & & & $.682 \dagger$ \\
\hline White background & $22(88)$ & $16(80)$ & \\
\hline Other background & $3(12)$ & $4(20)$ & \\
\hline Other Diagnoses & & & $.938 * *$ \\
\hline Yes & $6(24)$ & $5(25)$ & \\
\hline No & $19(76)$ & $15(75)$ & \\
\hline Previous Interventions & & & $.071 * *$ \\
\hline Speech Therapy & $14(56)$ & $9(45)$ & \\
\hline Other & $9(36)$ & $4(20)$ & \\
\hline None & $2(8)$ & $7(35)$ & \\
\hline Mean ADOS (severity) & $6.96(\mathrm{SD} 2.03)$ & $7.05(2.21)$ & $.766^{*}$ \\
\hline Mean SRS (total) & $115.44(\mathrm{SD} 26.12)$ & $126.0(28.73)$ & $.204 *$ \\
\hline Mean VABS-II (adaptive behaviour composite) & $68.40(\mathrm{SD} 14.04)$ & $69.20(14.76)$ & $.854 *$ \\
\hline \multicolumn{4}{|l|}{ Parent } \\
\hline Previous course attended & & & $.641 * *$ \\
\hline Yes & 12 & 11 & \\
\hline No & 13 & 9 & \\
\hline Family Make Up & & & $.716 \dagger$ \\
\hline Married/ living with partner & $20(80)$ & $17(85)$ & \\
\hline Single/ divorced & $5(20)$ & $3(15)$ & \\
\hline Parent Education & & & $.718 * *$ \\
\hline School leavers & $12(48)$ & $8(40)$ & \\
\hline A - Levels & $7(28)$ & $5(25)$ & \\
\hline University/Post-graduate & $6(24)$ & $7(35)$ & \\
\hline Parent Occupation & & & $.373 * *$ \\
\hline Full-time employed & $11(44)$ & $12(60)$ & \\
\hline Unemployed/ Other & $14(56)$ & $8(40)$ & \\
\hline Socioeconomic Status $\infty$ & $2.82(3.14 \mathrm{SD})$ & $2.39 \mathrm{M}(2.88 \mathrm{SD})$ & $.637 *$ \\
\hline
\end{tabular}

Note. $*^{*}$ test, $\dagger$ Fishers test, **Pearson's chi-square, $\infty$ Townsend Index of Deprivation 


\begin{tabular}{|c|c|c|c|c|c|c|c|c|c|}
\hline & \multicolumn{2}{|c|}{$\begin{array}{c}\text { Baseline } \\
\text { Mean (SD) }\end{array}$} & \multirow{2}{*}{$\begin{array}{c}\text { Baseline } \\
\text { equivalence } \\
p\end{array}$} & \multicolumn{2}{|c|}{$\begin{array}{c}\text { Time Point } 1 \\
\text { Mean (SD) }\end{array}$} & \multicolumn{2}{|c|}{$\begin{array}{l}\text { Time Point2 } \\
\text { Mean (SD) }\end{array}$} & \multicolumn{2}{|c|}{$\begin{array}{c}\text { Time Point } 3 \\
\text { Mean (SD) }\end{array}$} \\
\hline & Immediate & Delayed & & Immediate & Delayed & Immediate & Delayed & Immediate & Delayed \\
\hline Parent total RBQ-2 & $40.76(7.62)$ & $42.35(7.29)$ & .482 & $38.55(7.86)$ & $42.00(7.54)$ & $38.88(8.25)$ & $41.65(7.69)$ & $38.68(7.73)$ & $40.67(7.43)$ \\
\hline \multicolumn{10}{|l|}{ Parent factor RBQ-2 } \\
\hline Factor 1 & $9.56(2.89)$ & $10.65(2.08)$ & .164 & $9.32(2.58)$ & $10.35(2.13)$ & $9.44(2.57)$ & $9.30(1.89)$ & $9.44(2.65)$ & $9.45(2.14)$ \\
\hline Factor 2 & $14.44(4.11)$ & $14.65(3.83)$ & .862 & $13.60(3.58)$ & $14.10(3.70)$ & $13.36(4.28)$ & $14.70(3.77)$ & $13.40(4.07)$ & $14.22(3.60)$ \\
\hline Factor 3 & $15.24(2.89)$ & $15.10(3.16)$ & .878 & $14.15(3.37)$ & $15.40(2.89)$ & $14.24(3.09)$ & $15.85(3.28)$ & $14.24(2.89)$ & $15.35(2.91)$ \\
\hline Factor 4 & $7.28(2.11)$ & $7.75(2.47)$ & .495 & $6.40(1.98)$ & $7.75(2.51)$ & $7.24(2.11)$ & $8.10(2.07)$ & $6.84(1.75)$ & $7.46(2.34)$ \\
\hline \multicolumn{10}{|l|}{ Parent-child interactions } \\
\hline \multicolumn{10}{|l|}{ Child } \\
\hline Narrowed Interest & $4.60(4.40)$ & $4.70(5.57)$ & .918 & $4.56(4.25)$ & $4.14(5.79)$ & $4.35(3.88)$ & $5.04(6.04)$ & $4.54(4.74)$ & $7.45(8.64)$ \\
\hline Stereotyped behaviour ${ }^{\mathrm{b}}$ & $4.96(3.60)$ & $4.85(4.64)$ & .724 & $2.36(2.74)^{\mathrm{c}}$ & $4.83(4.17)^{\mathrm{c}}$ & $2.47(2.89)$ & $3.51(3.03)$ & $2.58(3.22)$ & $3.49(4.38)$ \\
\hline Sensory & $4.60(4.65)$ & $5.05(5.16)$ & .647 & $5.28(5.98)$ & $4.19(4.73)$ & $3.44(3.14)$ & $3.76(7.63)$ & $3.57(4.50)$ & $3.31(5.53)$ \\
\hline Motor & $2.24(3.05)$ & $3.20(4.70)$ & .449 & $3.00(3.45)$ & $1.75(1.92)$ & $2.88(3.98)$ & $1.35(1.87)$ & $3.43(5.88)$ & $1.85(2.50)$ \\
\hline Higher level words & $2.20(2.20)$ & $3.80(5.24)$ & .548 & $2.60(4.08)$ & $3.50(4.35)$ & $3.52(5.78)$ & $2.63(2.81)$ & $1.60(2.22)$ & $3.54(4.50)$ \\
\hline Lower level sounds & $4.20(6.01)$ & $3.10(3.20)$ & .839 & $3.44(5.35)$ & $3.15(4.62)$ & $2.64(2.91)$ & $1.95(1.99)$ & $2.41(3.33)$ & $2.02(1.89)$ \\
\hline \multicolumn{10}{|l|}{ Parent } \\
\hline Non-Intervening & $4.24(3.26)$ & $4.30(2.81)$ & .997 & $4.00(3.74)$ & $5.52(4.21)$ & $3.61(3.58)$ & $3.72(2.61)$ & $4.42(3.20)$ & $5.24(5.65)$ \\
\hline Preventing & $0.76(1.45)$ & $0.98(1.54)$ & .633 & $1.32(3.05)$ & $0.25(0.64)$ & $1.00(1.68)$ & $1.25(4.29)$ & $0.52(1.20)$ & $0.90(2.73)$ \\
\hline Engaging & $4.76(3.13)$ & $6.33(6.71)$ & .481 & $4.44(3.32)$ & $5.51(4.21)$ & $4.36(4.06)$ & $5.08(2.87)$ & $5.11(3.68)$ & $5.13(4.12)$ \\
\hline Distracting/developing $^{\mathrm{a}}$ & $7.12(5.31)$ & $7.83(4.57)$ & .518 & $6.64(5.87)$ & $6.98(4.70)$ & $7.19(3.88)^{\mathrm{c}}$ & $4.57(2.86)^{\mathrm{c}}$ & $5.54(4.51)$ & $6.65(5.49)$ \\
\hline Specific target RRB* & - & - & - & $3.83(1.08)$ & $4.38(1.23)$ & $3.46(1.38)$ & $3.98(1.58)$ & $3.43(1.59)$ & $4.28(1.34)$ \\
\hline Both target RRB* & - & - & - & $3.91(1.18)$ & $4.12(1.52)$ & $3.55(1.26)$ & $3.69(1.56)$ & $3.52(1.52)$ & $4.01(1.62)$ \\
\hline Factor 1 & - & - & - & $4.25(0.88)$ & $3.85(1.32)$ & $4.45(1.36)$ & $3.85(1.27)$ & $4.70(1.11)$ & $4.45(1.08)$ \\
\hline Factor 2 & - & - & - & $4.00(1.21)$ & $3.54(1.98)$ & $3.63(1.34)$ & $3.54(1.39)$ & $3.75(1.96)$ & $4.38(1.76)$ \\
\hline Factor 3 & - & - & - & $3.69(1.06)$ & $4.44(1.07)$ & $2.69(1.29)^{\mathrm{c}}$ & $4.59(1.49)^{\mathrm{c}}$ & $3.03(1.32)^{\mathrm{c}}$ & $5.03(0.81)^{\mathrm{c}}$ \\
\hline Factor 4 & - & - & - & $3.58(1.53)$ & $5.33(0.38)$ & $3.92(1.04)$ & $4.33(0.76)$ & $2.58(2.10)$ & $4.00(1.73)$ \\
\hline Teacher total RBQ-2 & $33.79(6.36)$ & $35.25(7.88)$ & .499 & $33.90(8.79)$ & $35.13(8.01)$ & $32.11(6.32)$ & $33.56(8.26)$ & $31.25(7.27)$ & $33.63(9.06)$ \\
\hline Parent self-efficacy $^{\mathrm{a}}$ & $2.94(1.06)$ & $2.54(0.79)$ & .173 & $3.38(0.97)^{\mathrm{c}}$ & $2.64(0.77)^{\mathrm{c}}$ & $3.41(1.07)^{\mathrm{c}}$ & $2.64(0.74)^{\mathrm{c}}$ & $3.61(0.91)^{c}$ & $2.40(0.70)^{\mathrm{c}}$ \\
\hline
\end{tabular}

*Note. Factor 1 - repetitive motor movement; Factor 2 - rigidity, adherence to routine and insistence on sameness; Factor 3 - preoccupation with restricted pattern of interest, limited play; Factor 4 - unusual sensory interests.

Specific behaviour =Level of improvement in the specific behaviour worked on in the immediate intervention group matched with a behaviour chosen from delayed groups that was the same category. Both behaviours = Level of improvement in both behaviours reported in both groups.

(a) significant interaction effects (b) significant effects of time within both immediate and delayed groups and (c) significant group difference. 


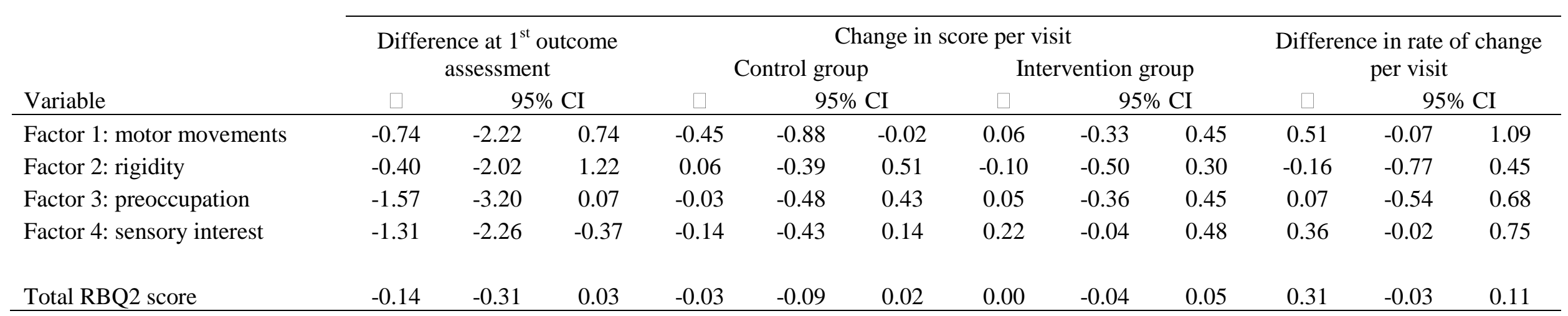




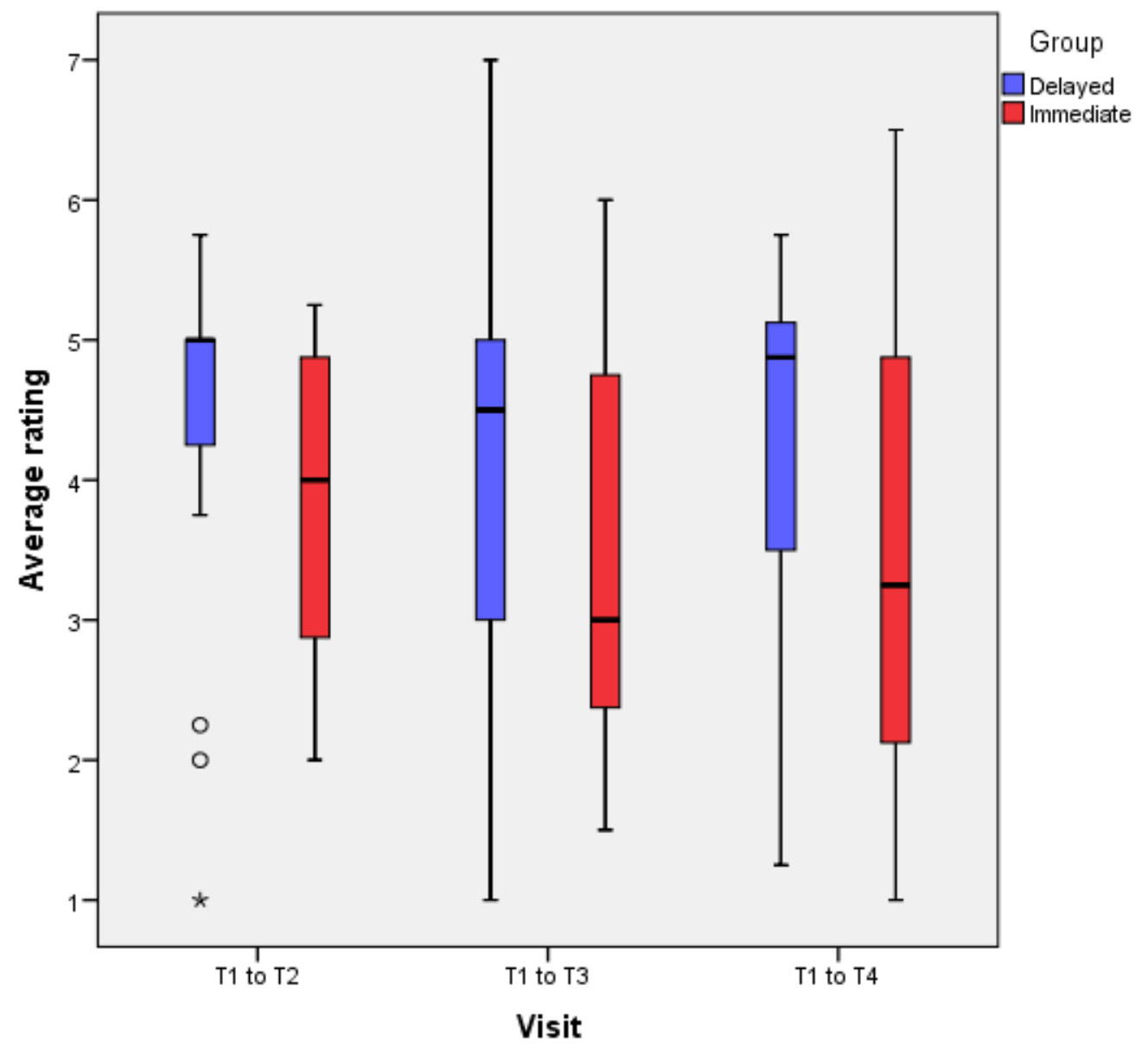




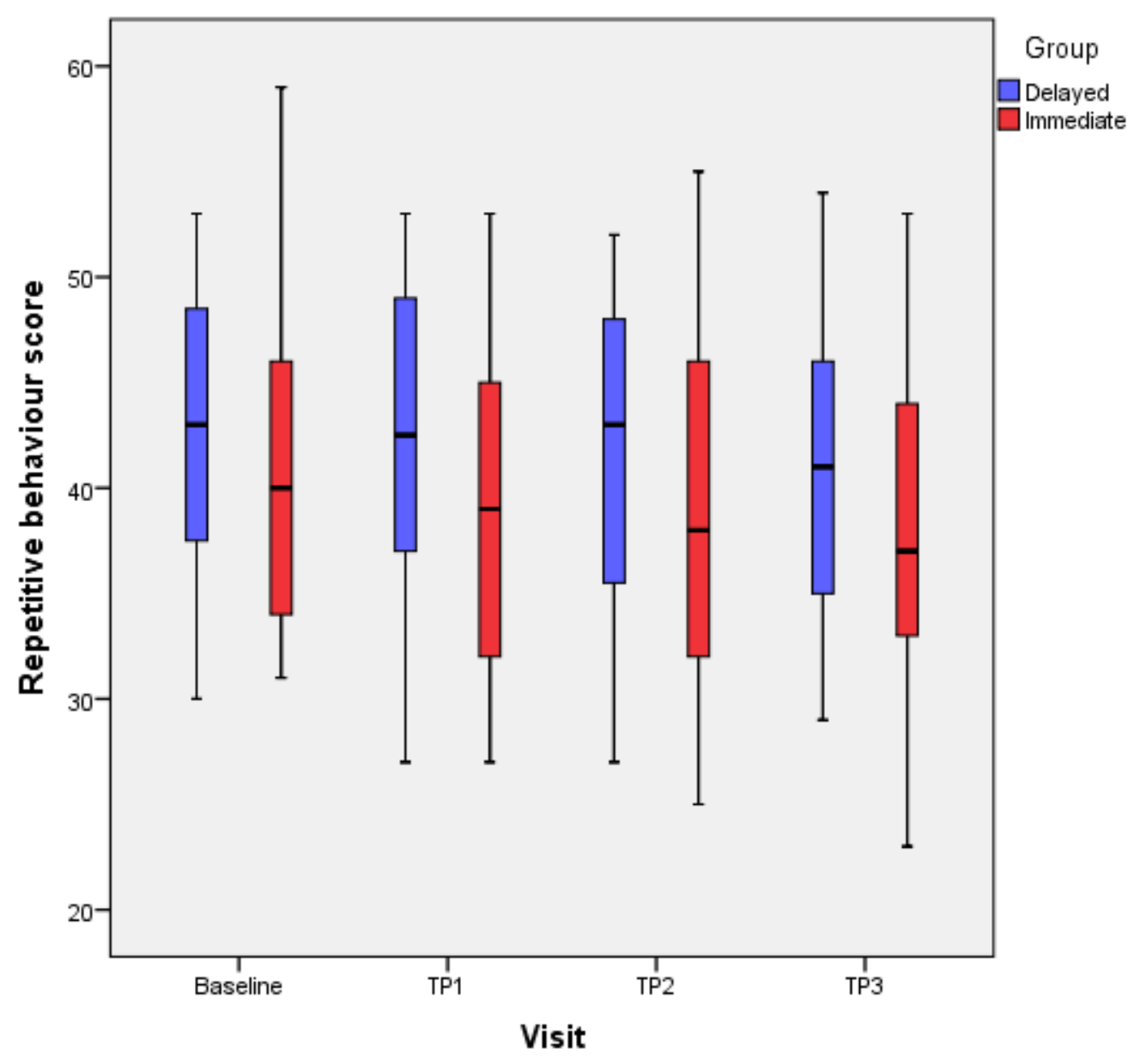




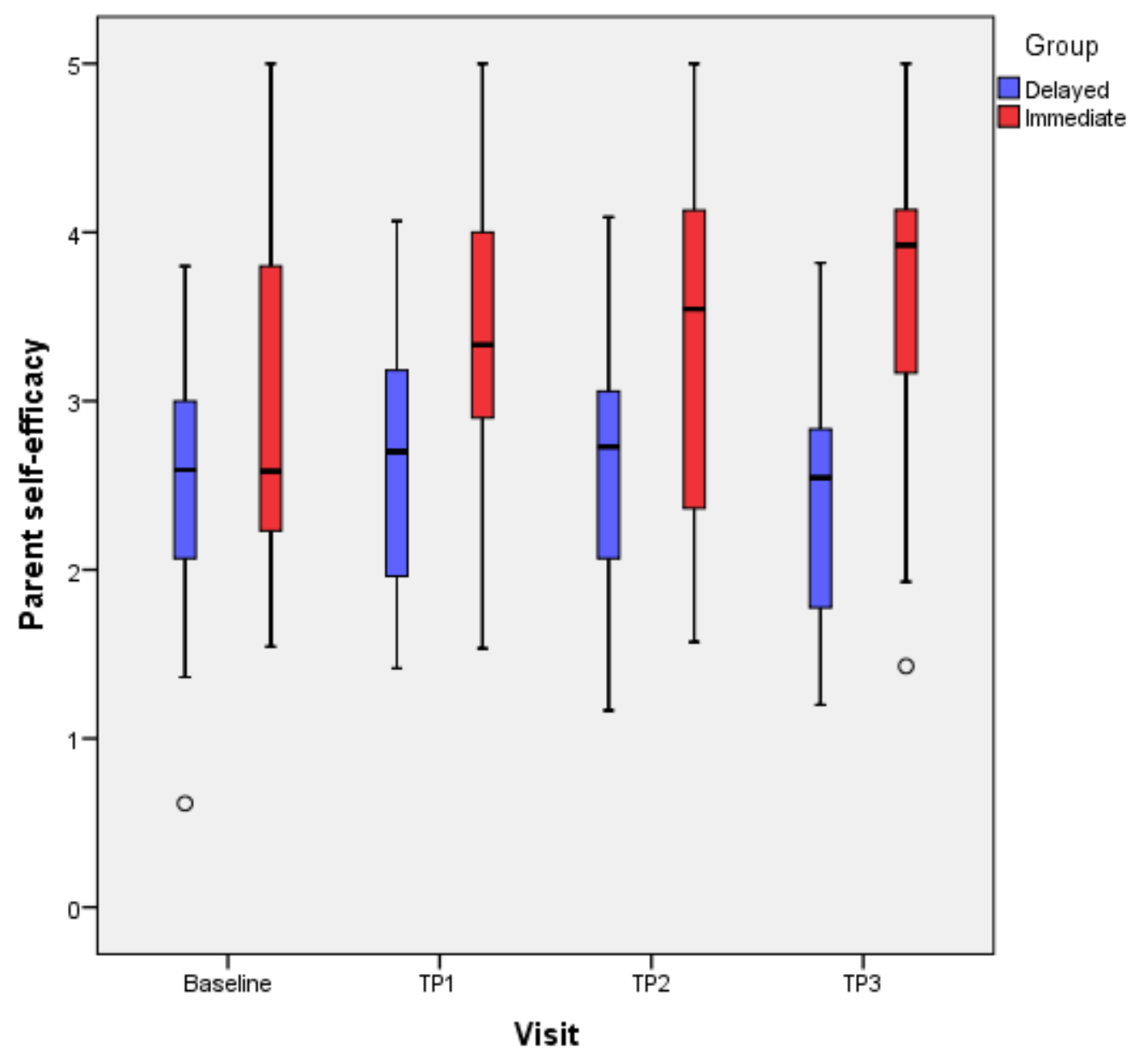


Figure Captions

Figure 1. CONSORT diagram

Figure 2. Change in target behaviour vignette

Figure 3. Parent rated total RBQ-2 by visit by group

Figure 4. Parent self-efficacy by visit by group. 